\title{
Keyword Index, Volume 90
}

\author{
16S rRNA 302
}

adaptation 107

additive variance 77,359

AFLP 10, 130, 268, 390

AFLPs 169, 326

Agavaceae 220

Agave deserti 220

Albizia julibrissin 187

allelic cluster 150

allozymes 64, 90, 98, 253, 451

AMOVA 253

annual Phlox 336

antibacterial peptides 382

apomixis 326

Arabidopsis lyrata 422

arrhenotoky 260

autopolyploid 308

autosomal inheritance 359

balancing selection 64

Bali cattle 10

banteng 10

Basque valleys 206

B chromosomes 122

Belgian blue cattle 350

bi-directional selection 336

Brassicaceae 422

Brassica napus 39

Canis familiaris 17

Canis lupus 17

character incompatibility 326

chemical defence 468

chiroptera 302

chromosomes 298

Cinnamomum insularimontanum 98

climate 397

clonal structure 25

coevolution 84

coevolution of genome conflict 122

columnar cacti 443

condition-dependent 359

Cydia pomonella 107

cytochrome $b 302$

cytoplasmic incompatibility 49, 157

Danaus chrysippus 236

demography 181, 282

diffusive dispersal 282

diploid 405

dispersal 64, 443

DNA 298

doubled haploid 33

double muscling 350

Drosophila 84, 157

Drosophila melanogaster 195

Drosophila simulans 49, 71

DXS548 206 ecology 302

effective pollen pool size 187

egg load 291

EM algorithm 459

endocellular bacteria 49, 157

endonucleases 298

endosperm traits 228

endozoochory 98

environmental variance 359

erucic acid 39

European corn borer 141

evolution of sex 260,397

eye 405

fatty acid 39

female-specific peptides 382

FISH 298, 405

fitness 371

fitness cost 107

flower size 336

FMR1 206

founder effect 282

four-way cross 459

FRAXAC1 206

fruit fly 365

$F_{\mathrm{ST}} 282$

Gekko 90

gene duplication 382

gene expression 405

gene flow 64, 90, 181, 212, 260, 268

genetic autocorrelation 25

genetic conflicts 157

genetic correlations 336, 359

genetic differentiation 212

genetic diversity 25, 181, 253

genetic interactions 84

genetic load 371

genetic structure 220, 268, 443

genetic variability 432

genetic variation 90

genome effect 150

genotyping errors 33

geographic mosaics 84

geographic parthenogenesis 397

grasshopper 377

habitat fragmentation 451

haploid 405

Hardy-Weinberg departures 432

heading 56

heat and cold tolerance 195

heritability 77,468

heterochromatin 377

heterosis 25

heterozygosity 90

heterozygote excess 236

hitchhiking 350

hsr-omega 195 
hybridism 236

hybridization 10, 17, 365

inbreeding 77

inbreeding depression 181, 308

insect immunity 382

iteratively reweighted least-squares method 228

karyotype 136

land snail 451

Laodelphax striatellus 71

latitudinal variation 195

lauraceae 98

life history 468

linkage disequilibrium 350, 432

linkage mapping 33

linkage maps 169, 390

locus order 33

\section{Madura 10}

mahogany 268

marker loci 247

mating system 308,443

mating time 365

maximum likelihood 459

maximum likelihood estimation 228

melanin 359

metapopulation 451

microinjection 71

microsatellites 17, 130, 268, 316, 397, 451

migration load 162

Mimulus guttatus 77

missing values 33

mitochondrial haplotypes 49

model 282

molecular evolution 382

mtDNA 17, 64

mutant mapping 390

mutational mechanism 150

mutations 371

myostatin 350

Myrica cerifera 25

Mytilus galloprovincialis 432

narrow-sense earliness 56

natural population 212

nucleolus organizer region 377

oil content 39

Ostrinia nubilalis 141

outcrossing rate 308

ovary 291

ovule selection 162

\section{parasitoids 84}

Paratya australiensis 64

parthenogenesis 130

partial incompatibility 422

PCR fingerprinting 253

Phlebotomus perniciosus 316

photoperiod sensitivity 56

phylogeny 302

phylogeography 302

pleiotropy 107
Pleurotus 253

pollen flow 162

pollen selection 162

polymorphism 195

polyploids 169,308

population genetics 253, 316

population structuration and differentiation 141

population structure 90

postglacial dispersal 316

protein polymorphisms 432

purging 371

Python molurus bivittatus 130

quantitative genetics 77,468

quantitative trait loci (QTL) 39

quantitative trait locus 228

quantitative trait locus analysis 56

RAPD 212

RAPDs 220

rate of evolution 336

rDNA 377

regulation 405

reproductive isolation 365

reproductive success 291

Reptilia 130

resistance 84

resistance management 107

S-alleles 422

sampling strategy 187

SAMPLs 169

sandfly 316

satDNA 377

satellite DNA 298

sawfly 468

SDMs 169

seed dispersal 98

seed flow 162

segregation distortion 33, 459

selection 107

sex chromosomes 136

sex-linked inheritance 359

sex-ratio 236

sexual and asexual reproduction 291

sexual forms 397

sexual isolation 236

sexual selection 371

SFLP 10

somatic mutation 247

Sonoran desert 443

Sonoran desert; speciation 220

Spain 316

spatial autocorrelation 25, 98

spatial genetic structure 98,451

spatial patterning 405

sperm retention 130

Spiroplasma 236

sporophytic self-incompatibility 422

SSR variation 150

Stenocereus gummosus 443

stratified dispersal 282

substitution lines 39

Swietenia macrophylla 268 
symbiosis 49,157

sympatric speciation 141

synaptonemal complex 136

Taraxacum 326

temporal variation 187

tephritidae 365

thelytoky 260

tradeoff 195

transgenic insecticidal maize 141

trees 247

triploid 228

Triticum dicoccoides 150

two-dimensional electrophoresis 432

TwoGener 187 unidirectional cytoplasmic incompatibility 71

Venturia canescens 260, 291

vernalization requirement 56

virulence 84

virus-like particle protein 291

wheat 56

wheat's progenitor 150

willow 169

Wolbachia 49, 71, 157

Y chromosome 17

Zea mays 122

zebrafish 136 